\title{
Sitagliptin for Elderly Patients Aged 75 Years or Older with Inadequately Controlled Type 2 Diabetes with Common Antidiabetes Treatments
}

\author{
Katsunori Suzuki', Yurie Mistuma', Takaaki Sato', Mariko Hatta ${ }^{2}$ \\ ${ }^{1}$ Division of Endocrinology and Metabolism, Saiseikai Niigata Daini Hospital, Niigata, Japan \\ ${ }^{2}$ Department of Nutrition, Saiseikai Niigata Daini Hospital, Niigata, Japan \\ Email: katsu-s@ngt.saiseikai.or.jp
}

Received 21 July 2015; accepted 20 September 2015; published 23 September 2015

Copyright (C) 2015 by authors and Scientific Research Publishing Inc.

This work is licensed under the Creative Commons Attribution International License (CC BY). http://creativecommons.org/licenses/by/4.0/

(c) (7) Open Access

\section{Abstract}

Background: The purpose of this study was to evaluate the effects of sitagliptin in elderly patients with type 2 diabetes aged 75 years or older versus those aged 65 - 74 years. Methods: Outpatients aged 65 years or older with type 2 diabetes who received sitagliptin at a dose of $50 \mathrm{mg}$ daily for 6 months were divided into two groups: those who were 75 years and older and those who were 65 74 years. The efficacy and safety were retrospectively evaluated by comparison of laboratory values before and after the administration of sitagliptin and by review of adverse events after treatment. Results: One hundred and twelve older patients with type 2 diabetes were studied. Six months after the initiation of sitagliptin, participants' hemoglobin A1c was significantly decreased by $1.09 \% \pm 0.8 \%$ in 65 - 74-year-olds (66 patients; mean age, $69.1 \pm 3.0$ years; mean HbA1c before administration, $8.4 \% \pm 0.8 \%$ ) and by $1.05 \% \pm 0.8 \%$ in patients 75 years or older (46 patients; mean age, $79.8 \pm 4.1$ years; mean HbA1c before administration, $8.5 \% \pm 0.7 \%$ ). There was no significant difference in hemoglobin A1c between the two groups. Furthermore, sitagliptin was well tolerated in both age groups. Conclusions: In elderly patients (75 years or older) with type 2 diabetes, the effect of sitagliptin was similar that in older patients (younger than 75 years) with type 2 diabetes.

\section{Keywords}

Elderly, Sitagliptin, Type 2 Diabetes Mellitus 


\section{Introduction}

According to the National Health and Nutrition Survey in Japan conducted in 2012, the number of people "strongly suspected to have diabetes" is rising in Japan. Those 65 years and older accounted for more than half of the patients studied [1]. Moreover, as the population ages, the number of patients aged 75 years or older with diabetes will also increase. However, an analysis [2] of large-scale clinical trials showed that only $0.6 \%$ of interventional trials in diabetes treatment specifically targeted this age group; $31 \%$ excluded patients older than 65 ; and almost all excluded those older than 75. Treatment of elderly patients with type 2 diabetes is challenging because of the high prevalence of comorbidities, use of polypharmacy, frailty, and the age-related reduction in pancreatic islet cell function [3]. Safety is therefore an important consideration for treatment, especially the avoidance of iatrogenic hypoglycemia which occurs frequently in elderly patients and can have severe consequences [4] [5]. Renal impairment is also very common in elderly patients with type 2 diabetes, which increases their risk for hypoglycemia and complicates treatment strategies [6]. The management of elderly patients with type 2 diabetes is further complicated by its clinical heterogeneity, i.e., very different disease durations and degrees of frailty, as well as the very limited availability of clinical trial data, especially in the very elderly subgroup [7].

The dipeptidyl peptidase-4 (DPP4) inhibitors, a new class of oral antidiabetic agents, could be particularly promising for the treatment of older patients [8] [9]. Sitagliptin, a member of this class, has been studied in a large clinical program, showing good overall safety and tolerability and improving glycemic control when given as monotherapy or combination therapy [10]-[14]. A very low incidence of hypoglycemia has consistently been reported with sitagliptin across the disease spectrum [15]-[20]. In addition, we consider that sitagliptin is effective for the treatment of type 2 diabetes in elderly patients because it is taken once daily and therefore adherence to the treatment is favorable.

The aim of this study was to investigate the glucose-lowering efficacy, safety, and tolerability of sitagliptin in patients aged 65 - 74 years versus those 75 years and older with type 2 diabetes whose glycemic control was inadequate on other common glucose-lowering drugs.

\section{Methods}

\subsection{Study Design and Patients}

Among patients aged 65 years and older with type 2 diabetes being treated at our diabetes clinic on an outpatient basis, those who received sitagliptin once daily at a dose of $50 \mathrm{mg}$ ( $25 \mathrm{mg}$ when estimated glomerular filtration rate [eGFR] was below $30 \mathrm{~mL} / \mathrm{min}$ per $1.73 \mathrm{~m}^{2}$ ), and who were treated for at least 6 months were examined. Changes in hemoglobin A1c (HbA1c) before and after treatment with sitagliptin were retrospectively analyzed. The patients were divided into two groups: those 75 years or older and those $65-74$ years. The difference in the effect of sitagliptin between these two groups was assessed. The observation period was from January 2010 to January 2014. Medical records were examined for dosages administered and adverse events (AEs). Exclusion criteria included add on other antidiabetic agents or drugs affecting plasma glucose or changes in the previously use of other antidiabetic agents and in instructions for diet or exercise therapy during the study period.

\subsection{Assessments}

HbA1c, body weight, sitting systolic and diastolic blood pressure and common laboratory parameters were measured periodically during the study period. All adverse events (AEs) were recorded and assessed by the investigator with regard to severity and possible relationship to the study medication.

Glomerular filtration rate (GFR) was estimated by the Modification of Diet in Renal Disease (MDRD) method and data in the group of patients with severe renal impairment (eGFR $<30 \mathrm{~mL} / \mathrm{min} / 1.73 \mathrm{~m}^{2}$ ) are presented (Table 1). Confirmed hypoglycemia was defined as symptoms suggestive of low blood glucose confirmed by self-monitored blood glucose (SMBG) measurement $<3.1 \mathrm{mmol} / \mathrm{L}$ plasma glucose equivalent. Severe hypoglycemia was defined as any episode requiring the assistance of another party.

\subsection{Data Analysis}

The data were summarized by mean values and standard deviations. Statistical analysis was performed using 
Table 1. Baseline characteristics by age groups.

\begin{tabular}{|c|c|c|c|}
\hline & $\begin{array}{c}65-74 y \\
(n=66)\end{array}$ & $\begin{array}{c}\geq 75 \mathrm{y} \\
(\mathrm{n}=46)\end{array}$ & $P$-value \\
\hline Age (y) & $69.1 \pm 3.0$ & $79.8 \pm 4.1$ & $<0.001$ \\
\hline Male, No. (\%) & $38(57.5)$ & 27 (58.6) & 0.999 \\
\hline Body weight (kg) & $61.3 \pm 10.8$ & $58.0 \pm 10.2$ & 0.196 \\
\hline BMI $\left(\mathrm{kg} / \mathrm{m}^{2}\right)$ & $24.3 \pm 3.8$ & $23.8 \pm 3.3$ & 0.453 \\
\hline Duration of Diabetes (y) & $14.4 \pm 7.3$ & $16.1 \pm 8.8$ & 0.274 \\
\hline HbAc1 (\%) & $8.43 \pm 0.8$ & $8.49 \pm 0.7$ & 0.725 \\
\hline SBP (mmHg) & $126.5 \pm 12.5$ & $123.8 \pm 9.8$ & 0.223 \\
\hline DBP (mmHg) & $72.9 \pm 9.9$ & $69.0 \pm 10.3$ & 0.051 \\
\hline eGFR (ml/min/1.73 m²) & $74.6 \pm 22.2$ & $67.0 \pm 19.3$ & 0.068 \\
\hline \multicolumn{4}{|l|}{ Complication $^{\dagger}$} \\
\hline Hypertension & $41(62.1)$ & $34(73.0)$ & 0.224 \\
\hline Dyslipidemia & $32(48.4)$ & $19(41.3)$ & 0.563 \\
\hline Chronic Heart failure & $5(7.5)$ & $1(2.1)$ & 0.398 \\
\hline OMI & $5(7.7)$ & $8(17.3)$ & 0.138 \\
\hline $\mathrm{AF}$ & $1(1.5)$ & $2(4.3)$ & 0.567 \\
\hline ASO & $1(1.5)$ & $2(4.3)$ & 0.567 \\
\hline Stroke & $1(1.5)$ & $0(0.0)$ & 0.999 \\
\hline \multicolumn{4}{|l|}{ Other $\mathrm{OHA}^{\ddagger}$} \\
\hline Metformin & $47(71.2)$ & $22(47.8)$ & 0.018 \\
\hline SU & 31 (46.9) & $27(58.7)$ & 0.252 \\
\hline Pioglitazone & $6(9.0)$ & $7(15.2)$ & 0.376 \\
\hline$\alpha \mathrm{GI}$ & $2(3.0)$ & $2(4.3)$ & 0.999 \\
\hline Insulin & 15 (22.7) & $13(28.2)$ & 0.515 \\
\hline
\end{tabular}

Unless indicated otherwise, data are shown as the mean \pm SD. Values show the difference compared between two groups (two-tailed Student's paired or unpaired t-test and Fisher's exact test). ${ }^{\dagger}$ All complications are expressed as No. (\%); ${ }^{\ddagger}$ Oral hypoglycemic agents expressed as No. (\%); Abbreviations: BMI: body mass index; SBP: systolic blood pressure; DBP: diastolic blood pressure; eGFR: estimated glomerular filtration rate; OMI: old myocardial infarction; AF: atrial fibrillation; ASO: arteriosclerosis obliterans; OHA: oral hypoglycemic agents; SU: sulfonylurea; $\alpha \mathrm{GI}$ : $\alpha$-glucosidase inhibitor.

StatView version 5.0 for Windows (SAS Institute, City, NC, USA). The significance of differences between discrete variable data was analyzed by Fisher's exact test as appropriate. Differences between two variables were evaluated by two-tailed Student's paired or unpaired t-test as appropriate. A two-sided significance level of 5\% was used. All HbA1c values are shown as National Glycohemoglobin Standardization Program values.

\subsection{Ethics and Good Clinical Practice}

All study participants provided written informed consent. This study was conducted according to the principals expressed in the Declaration of Helsinki. Informed consent was obtained from each subject after full explanation of the purpose, nature, and risks of all procedures used. The study protocol was approved by the ethical review committee of Saiseikai Niigata Daini Hospital. 


\section{Results}

As a result of screening during the above observation period, 142 patients were recruited for the study. Among them, 30 patients were excluded for the reasons mentioned in the study design section, and 112 patients with type 2 diabetes (65 men and 47 women; mean age, $73.5 \pm 6.3$ years; BMI, $24.1 \pm 3.6 \mathrm{~kg} / \mathrm{m}^{2}$; disease duration, $15.1 \pm 8.0$ years; baseline HbA1c, 8.4\% $\pm 0.8 \%$ ) were included in the study (Figure 1). After 6 months of treatment with sitagliptin, a significant decrease in HbA1c, by $1.07 \% \pm 0.8 \%(P<0.001)$, was observed in all patients. Table 1 shows the characteristics for patients 65 - 74 years old and those 75 years or older. The former group consisted of 66 patients. The mean age was $69.1 \pm 3.0$ years old (range, $65-74$ ). Baseline HbA1c was 8.4 $\pm 0.8 \%$. The latter group consisted of 46 patients. The mean age was $79.8 \pm 4.1$ years old (range, $75-92$ ). Baseline $\mathrm{HbA1c}$ was $8.5 \% \pm 0.7 \%$. The proportion of patients who used metformin at baseline was significantly lower in the latter group. Figure 2 shows changes in HbA1c in the two groups. Both groups demonstrated a significant decrease 3 months after the start of treatment with sitagliptin, which persisted up to 6 months. Changes

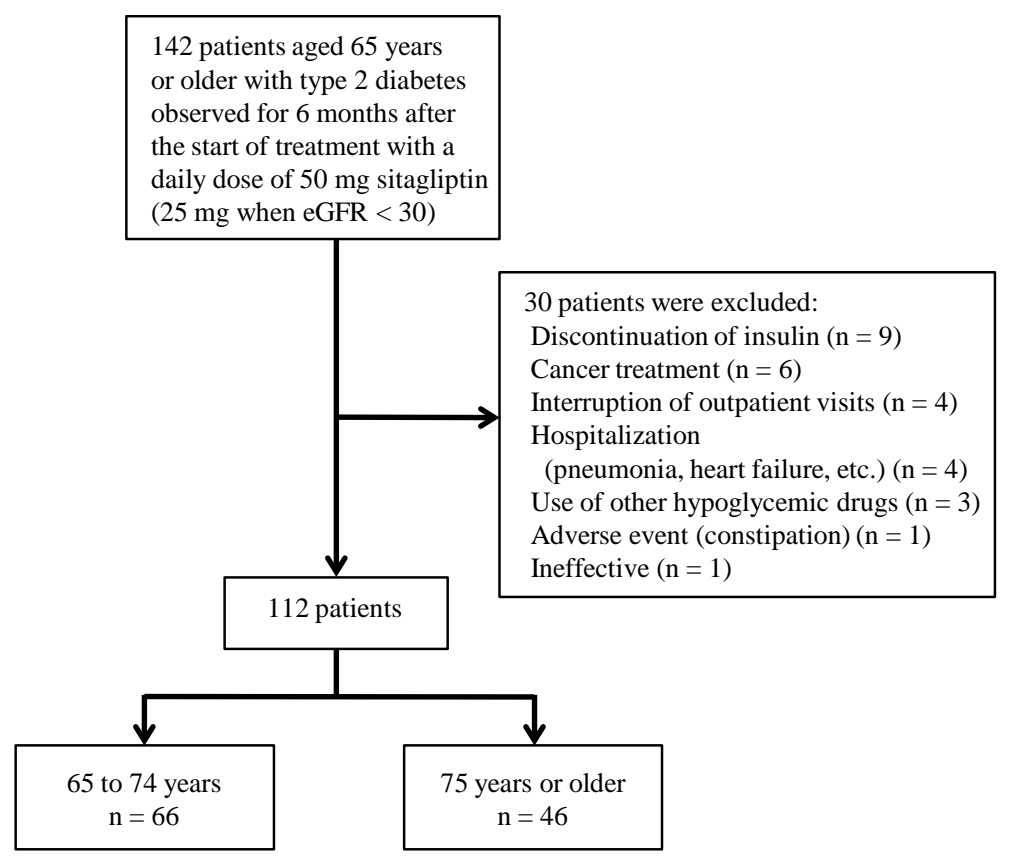

Figure 1. Trial profile.

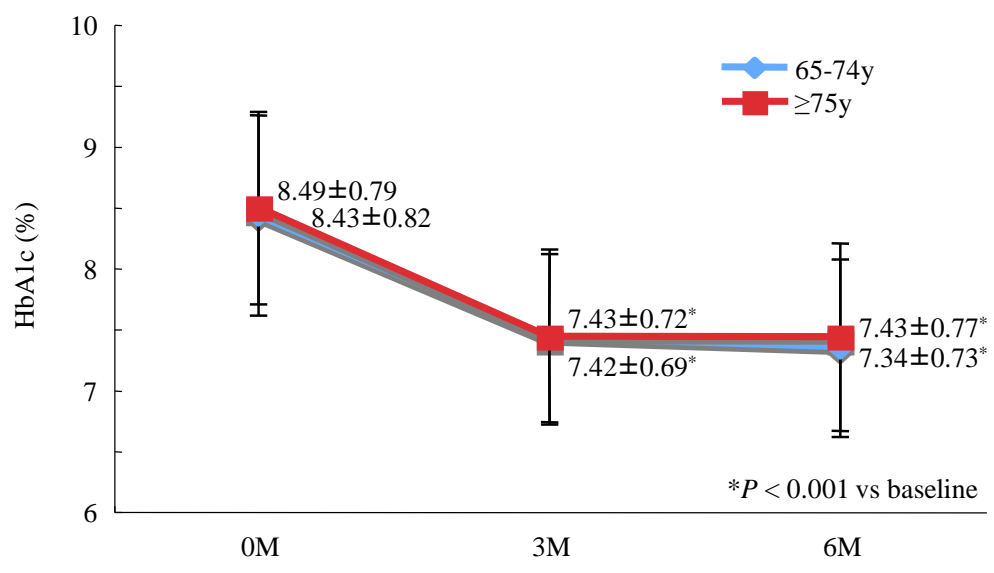

Data are showed as the mean $\pm \mathrm{SD}$. ${ }^{*} \mathrm{p}<0.001$ compared with baseline. No significant difference was observed between the two groups at baseline, 3 months and 6 months.

Figure 2. Change in the HbA1c levels from baseline at six-month intervals. 
in HbA1c were thus similar among the two groups represented by a decrease of $1.09 \% \pm 0.8 \%$ and $1.05 \% \pm 0.8 \%$ for the 65 - 74-year-old and the 75 years or older groups, respectively, indicating a significant decrease from baseline in each group. No significant difference was observed between the two groups (Figure 3).

The change in HbA1c in relation to previous hypoglycemic agents used at baseline is shown in Figure 4. HbA1c was compared for patients previously treated with the following concomitant drugs: sulfonylurea (SU) alone; metformin alone; both SU and metformin; metformin and insulin; and insulin alone. HbA1c decreased significantly in all groups and no significant difference was observed between the two age groups. Next, change in HbA1c is presented versus the duration of diabetes in Figure 5. HbA1c was compared based on duration of disease: less than 5 years; 5 to 14 years; and 15 years or longer. HbA1c was significantly decreased in all groups. No significant difference was detected between the two age groups. Figure 6 shows the change in HbA1c versus

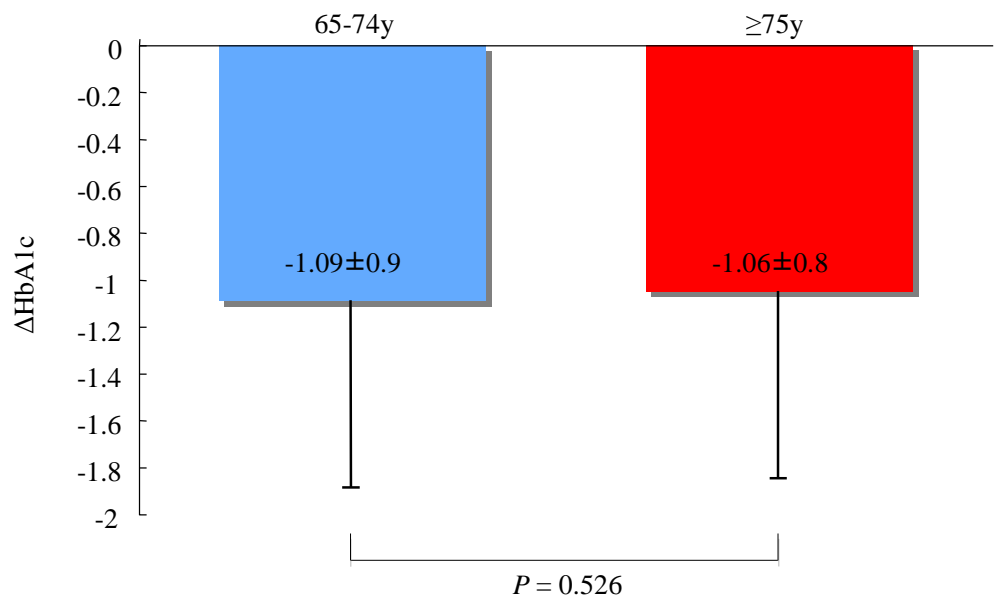

Data are showed as the mean \pm SD.

No significant difference was observed between the two groups.

Figure 3. Adjusted mean changes in HbA1c from baseline to six months.

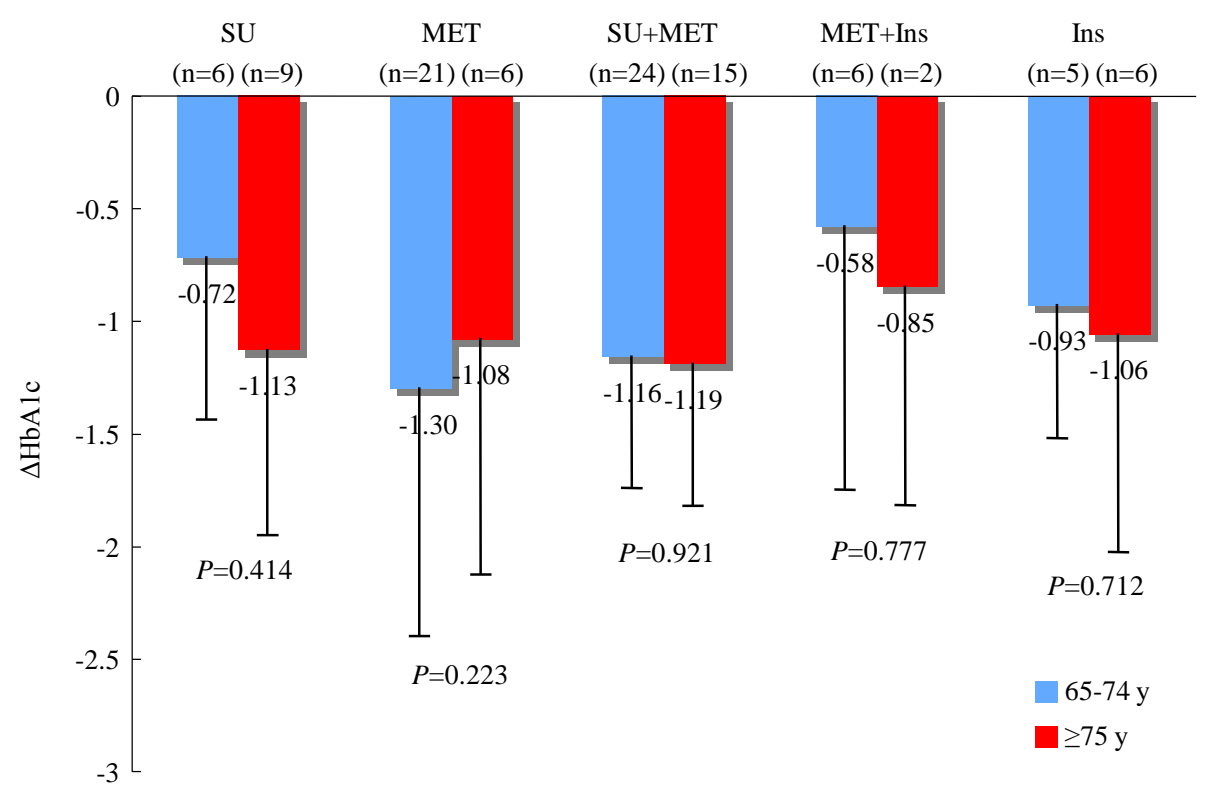

Data are showed as the mean \pm SD. HbA1c decreased significantly in all groups and no significant difference was observed between the two age groups. SU: sulfonylurea alone; MET: metformin alone; SU+MET: both SU and metformin; MET+INS: metformin and insulin; and Ins: insulin alone.

Figure 4. Change in HbA1c versus previous therapeutic agent at baseline. 


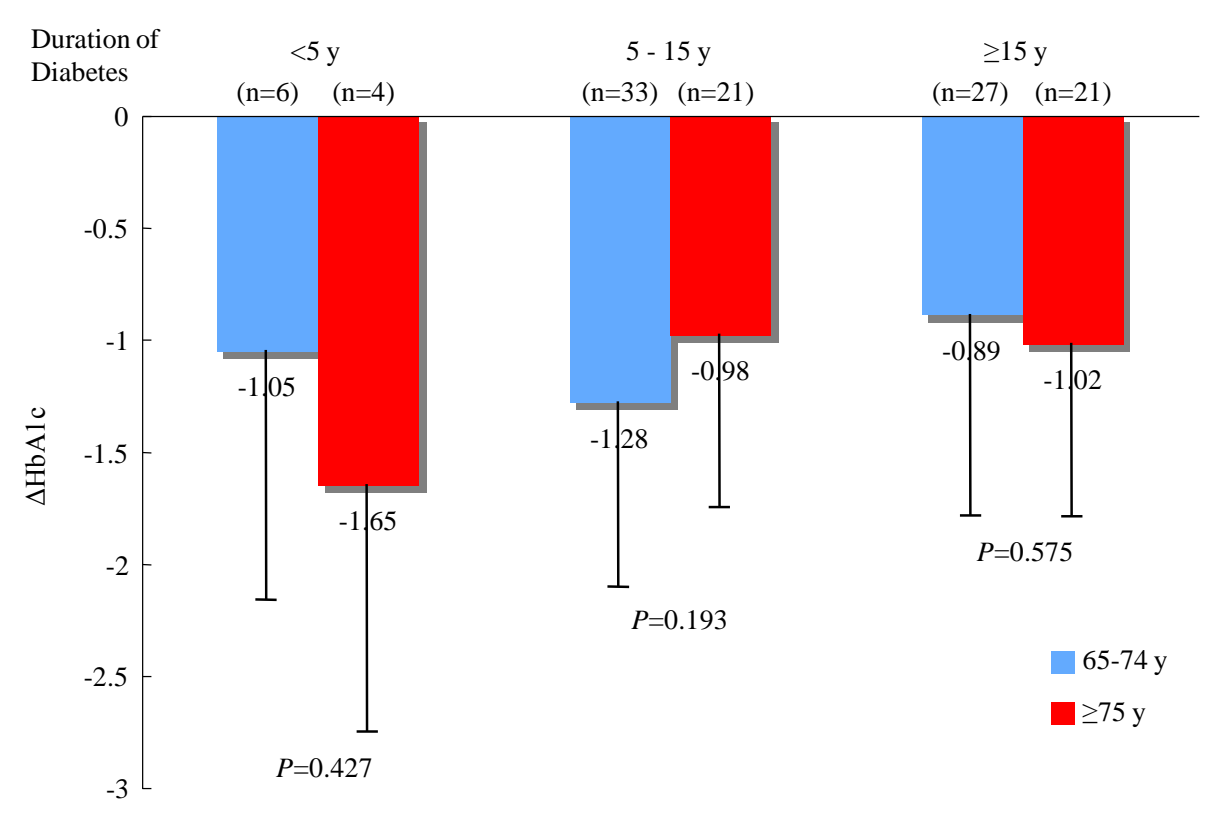

Data are showed as the mean \pm SD. HbA1c decreased significantly in all groups and no significant difference was observed between the two age groups.

Figure 5. Decrease in HbA1c six months after addition of sitagliptin versus duration of diabetes.

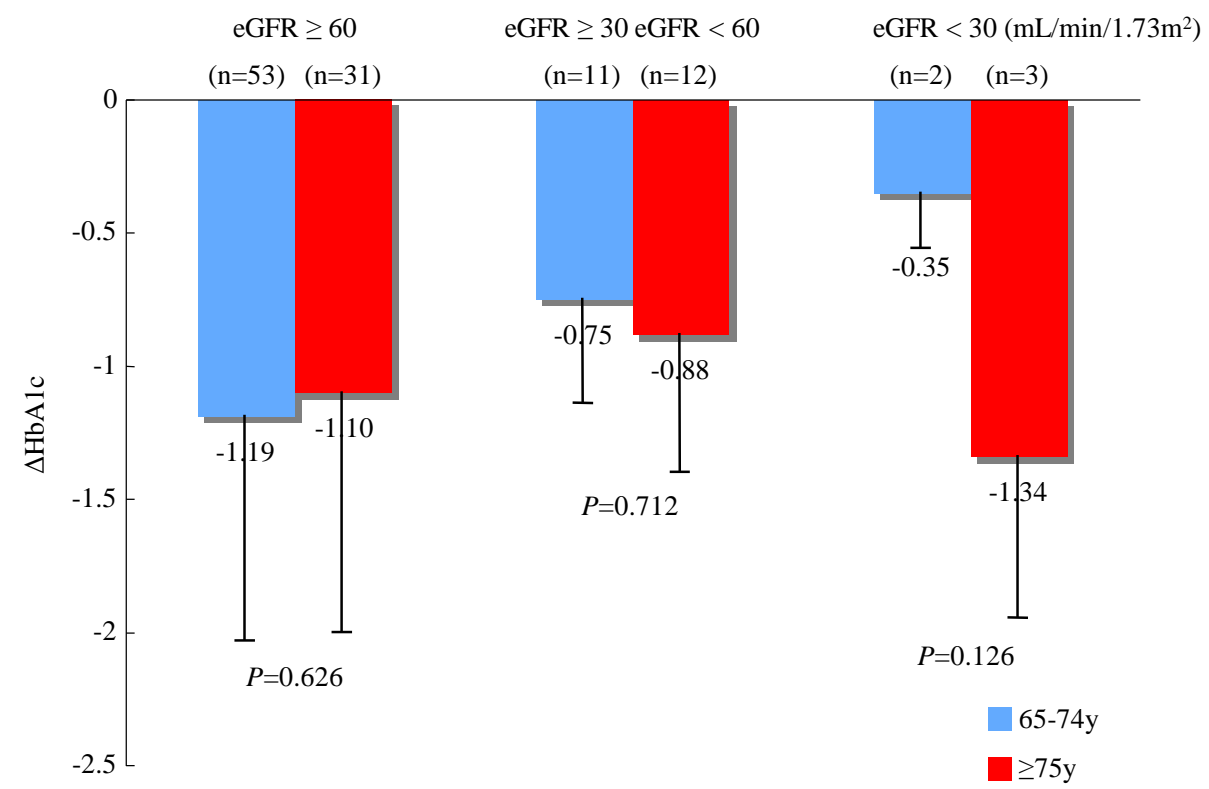

Data are showed as the mean $\pm \mathrm{SD}$. HbA1c decreased significantly in all groups and no significant difference was observed between the two age groups. eGFR: estimated glomerular filtration rate;

\section{Figure 6. Change in HbA1c versus baseline eGFR.}

baseline eGFR. HbA1c was examined for patients whose eGFR was $<30 \mathrm{~mL} / \mathrm{min} / 1.73 \mathrm{~m}^{2}$, patients whose eGFR was $\geq 30 \mathrm{~mL} / \mathrm{min} / 1.73 \mathrm{~m}^{2}$ and $<60 \mathrm{~mL} / \mathrm{min} / 1.73 \mathrm{~m}^{2}$, and patients whose eGFR was $>60 \mathrm{~mL} / \mathrm{min} / 1.73 \mathrm{~m}^{2}$. In all groups, HbA1c decreased significantly. There was no significant difference between the two age groups.

Table 2 compares changes in body weight and blood pressure between the two groups. Body weight and systolic blood pressure did not significantly change in either group. In patients aged 75 years or older, diastolic 
Table 2. Changes in body weight and systolic and diastolic blood pressure in the two groups.

\begin{tabular}{|c|c|c|c|c|c|}
\hline BW (kg) & 0 Months & 3 Months & 6 Months & $\begin{array}{l}\text { Adjusted mean change } \\
\text { from baseline at } \\
6 \text { Months }\end{array}$ & $\begin{array}{c}P \text {-value at } 6 \mathrm{M} \\
\text { vs. Baseline }\end{array}$ \\
\hline $65-74 y$ & $61.3 \pm 10.9$ & $61.3 \pm 10.9$ & $61.3 \pm 11.0$ & $0.024 \pm 0.00$ & 0.916 \\
\hline$\geq 75 y$ & $58.0 \pm 10.2$ & $58.1 \pm 10.2$ & $58.2 \pm 10.4$ & $0.132 \pm 0.00$ & 0.622 \\
\hline \multicolumn{6}{|c|}{$P=0.124$ between two groups. } \\
\hline SBP (mmHg) & 0 Months & & 6 Months & $\begin{array}{l}\text { Adjusted mean change } \\
\text { from baseline at } \\
6 \text { Months }\end{array}$ & $\begin{array}{c}P \text {-value at } 6 \mathrm{M} \\
\text { vs. Baseline }\end{array}$ \\
\hline $65-74 y$ & $126.5 \pm 12.4$ & & $126.1 \pm 14.3$ & $-0.409 \pm 11.6$ & 0.766 \\
\hline$\geq 75 y$ & $123.8 \pm 9.83$ & & $123.8 \pm 12.2$ & $0.00 \pm 10.6$ & 1.000 \\
\hline \multicolumn{6}{|c|}{$P=0.376$ between two groups. } \\
\hline DBP (mmHg) & 0 Months & & 6 Months & $\begin{array}{l}\text { Adjusted mean change } \\
\text { from baseline at } \\
6 \text { Months }\end{array}$ & $\begin{array}{c}P \text {-value at } 6 \mathrm{M} \\
\text { vs. Baseline }\end{array}$ \\
\hline $65-74 y$ & $72.9 \pm 9.9$ & & $72.9 \pm 10.9$ & $-0.03 \pm 11.0$ & 0.982 \\
\hline$\geq 75 y$ & $69.1 \pm 10.3$ & & $66.9 \pm 12.1$ & $-2.10 \pm 13.5$ & 0.296 \\
\hline
\end{tabular}

$P=0.008$ between two groups.

Data are shown as the mean \pm SD. Values show the difference compared between two groups. (two-tailed Student's paired or unpaired t-test). BW: body weight; SBP: systolic blood pressure; DBP: diastolic blood pressure.

blood pressure was slightly lowered and showed a significant difference at 6 months from the values in patients younger than 75 years.

There were few AEs. Constipation was observed in 2 patients (1 in each age group) and mild hypoglycemia in 2 patients (a patient treated with SU in those younger than 75 and a patient treated with insulin in those 75 years or older). No serious AEs were observed in either group. Sitagliptin was well tolerated.

\section{Discussion}

The present study demonstrates that, in elderly patients aged 75 years or older with type 2 diabetes whose plasma glucose had been insufficiently controlled by other hypoglycemic agents, sitagliptin improved plasma glucose level as effectively as in those patients aged 65 - 74 with poorly controlled type 2 diabetes. Furthermore, the drug was well tolerated. Review of the literature demonstrates that patients aged 75 years or older with type 2 diabetes have generally been excluded from clinical trials, even though they account for a large and growing proportion of patients with type 2 diabetes.

Perhaps one reason for the exclusion from such trials is concern about the risk of hypoglycemia, which poses a problem in drug therapy in older patients with diabetes. Hypoglycemia not only increases the risk of falls and fractures, but also possibly causes impairment of cognitive function [21] [22]. Authors of the Action to Control Cardiovascular Risk in Diabetes (ACCORD) Trial pointed out the relationship between severe hypoglycemia and higher mortality, specifically cardiovascular deaths, seen significantly more frequently in the intensive glucose control group than in the standard glucose control group [23]. This highlights the importance of prevention of hypoglycemia. The reasons for frequent hypoglycemia in elderly patients include: 1) slowed metabolism and excretion of drugs related to aging, 2) impairment of autonomic function, 3) decreased or unstable glucose intake, and 4) poor adherence to treatment due to impaired cognitive function.

The most important age-related change in pharmacokinetics occurs in the kidney. The weight of the kidney decreases with aging. Renal blood flow is speculated to be reduced by $1 \mathrm{~mL} /$ minute/year after the age of 30, resulting in a linear age-related decrease in eGFR starting in one's 40s. Additionally, many drugs are mainly metabolized in the liver. With aging, the weight of the liver also decreases and its metabolic capacity is reduced. These age-related changes delay the metabolism and excretion of drugs and result in an increased concentration 
of drugs in the blood, which can lead to significant decreases in plasma glucose as well as other adverse drug reactions. Moreover, impairment of autonomic function increases the risk for sudden dysfunction of the central nervous system, which is responsible for subjective symptoms that warn of decreases in plasma glucose level, rendering the patient unaware of hypoglycemia. Thus, hypoglycemia is more likely to become severe. Furthermore, glucose intake may be greatly reduced due to seasonal changes and changes in environment for many elderly patients. In such cases, hypoglycemia can easily occur in patients treated with SUs, unless they are told how to prevent it. Impairment of cognitive function increases the risk of duplicate dosing of drugs as well as errors in choosing the correct insulin formulation and administering the correct dosage. Iwakura et al. raised advanced age, impaired renal function, use of SUs and insulin, and low HbA1c as factors that increase the risk of severe hypoglycemia [24].

Because oral hypoglycemic agents directly affect glycemic control, the prescription instructions need to be as simple as possible in order to improve adherence to the treatment. When glycemic control is gradually worsened, prescribing physicians should remember to confirm whether or not these drugs are being taken properly before increasing the dose or adding new oral hypoglycemic agents. It is often difficult to confirm this by talking only with patients. Discussion with family members is also important.

DPP-4 inhibitors are classified as insulin secretagogues. However, they promote insulin secretion only when plasma glucose level is high, and therefore, they do not frequently cause hypoglycemia when used as oral monotherapy. This is the greatest advantage of these drugs for use in elderly patients. However, the risk of hypoglycemia is increased in concomitant use with other drugs, especially SUs or insulin. In addition, DPP-4 inhibitors are taken once or twice a day, which is expected to help maintain adherence to the treatment. When renal function is impaired, these drugs can be used by adjusting the dose (although dose adjustment is unnecessary for linagliptin and teneligliptin). Thus, we speculate that these drugs can be used relatively safely in elderly patients. Among DPP-4 inhibitors, the outcome of administration of vildagliptin and linagliptin has been, in particular, examined in more elderly subjects with diabetes than others. A meta-analysis of studies on vildagliptin showed that the improvement in HbA1c as a result of using this drug was comparable in patients aged 65 years or older and patients younger than 65 years. Fasting plasma glucose and body weight were significantly and markedly decreased in the group of patients aged 65 years or older [25]. Moreover, Schweizer et al. evaluated this drug in even older patients, those aged 75 years or older (mean age, 76.9 years; range, 75 - 84 years) and confirmed it to be effective and well tolerated [26]. The subjects of the present study were even older than those of Schweizer and colleagues, being 79.8 years old on average, ranging from 75 - 92 years old. The study by Schweizer et al. was a meta-analysis of Phase II and III clinical studies, and most participants were healthy enough to be eligible for participation in such clinical studies. In contrast, our subjects were recruited from clinical practice, and therefore, we consider our data to be a better reflection of actual treatment. As far as we know, the present study is the first report showing that sitagliptin, the least examined DPP-4 inhibitor, in elderly patients with type 2 diabetes is as effective and as well tolerated as others in this class.

Linagliptin was shown to significantly decrease HbA1c by $-0.64 \%$ compared with placebo and to be as safe and well tolerated as placebo in a study in which linagliptin or placebo was added to monotherapy with metformin, SU, or long-acting insulin, or to a combination of these drugs prescribed to patients aged 70 years or older [27]. Our study also demonstrates that sitagliptin is useful and safe when it is added to treatment with other hypoglycemic agents. Our study further shows its efficacy in elderly patients with diabetes aged 75 years or older with any renal dysfunction.

One major limitation of the present study is its retrospective design and the limited number of patients studied. Almost all of the patients received add-on therapy; the number of drug naïve patients was limited $(n=9)$. In addition, the duration of the study may be another limitation because the extent of the glycemic response may not have been fully elucidated over this time frame. Furthermore, other markers, such as markers for assessment of $\beta$-cell function in homeostatic models, and basal active GLP-1 levels, which could have contributed to the HbA1c reduction with sitagliptin, were not measured in the present study. Therefore, increased numbers of subjects and more detailed clinical parameters are needed in the future to avoid inappropriate extrapolation of the data.

The present study shows that sitagliptin added to existing glucose-lowering drugs is well tolerated, weightneutral, and improves glycemic control in patients with type 2 diabetes aged 75 years or older (with characteristics typical of those encountered in clinical practice), similar to those less than 75 years old, without increasing the risk of AEs. 


\section{Acknowledgements}

The authors would like to acknowledge the patients and staff at participating sites for this study. The authors thank Forte (https://www.forte-science.co.jp) for English language review.

\section{References}

[1] Japan Ministry of Health, Labor and Welfare: The 2012 National Health and Nutrition Survey in Japan. http://www.mhlw.go.jp/stf/houdou/0000032074.html

[2] Lakey, W.C., Barnard, K., Batch, B.C., Chiwell, K., Tasneem, A. and Green, J.B. (2013) Are Current Clinical Trials in Diabetes Addressing Importance Issues in Diabetes Care? Diabetologia, 56, 1226-1235. http://dx.doi.org/10.1007/s00125-013-2890-4

[3] Fravel, M.A., McDanel, D.L., Ross, M.B., Moores, K.G. and Starry, M.J. (2011) Special Considerations for Treatment of Type 2 Diabetes Mellitus in the Elderly. American Journal of Health-System Pharmacy, 68, 500-509. http://dx.doi.org/10.2146/ajhp080085

[4] Kirkman, M.S., Briscoe, V.J., Clark, N., et al. (2012) Diabetes in Older Adults. Diabetes Care, 35, 2650-2664. http://dx.doi.org/10.2337/dc12-1801

[5] Sinclair, A., Morley, J.E., Rodriguez-Mañas, L., et al. (2012) Diabetes Mellitus in Older People: Position Statement on Behalf of the International Association of Gerontology and Geriatrics (IAGG), the European Diabetes Working Party for Older eople (EDWPOP), and the International Task Force of Experts in Diabetes. Journal of the American Medical Directors Association, 13, 497-502. http://dx.doi.org/10.1016/j.jamda.2012.04.012

[6] Inzucchi, S.E., Bergenstal, R.M., Buse, J.B., et al., the American Diabetes Association (ADA), and the European Association for the Study of Diabetes (EASD) (2012) Management of Hyperglycemia in Type 2 Diabetes: A PatientCentered Approach: Position Statement of the American Diabetes Association (ADA) and the European Association for the Study of Diabetes (EASD). Diabetes Care, 35, 1364-1379. http://dx.doi.org/10.2337/dc12-0413

[7] Halimi, S., Raccah, D., Schweizer, A. and Dejager, S. (2010) Role of Vildagliptin in Managing Type 2 Diabetes Mellitus in the Elderly. Current Medical Research and Opinion, 26, 1647-1656. http://dx.doi.org/10.1185/03007995.2010.485881

[8] Mathieu, C. and Bollaerts, K. (2007) Antihyperglycaemic Therapy in Elderly Patients with Type 2 Diabetes: Potential Role of Incretinmimetics and DPP-4 Inhibitors. International Journal of Clinical Practice, 154, 29-37. http://dx.doi.org/10.1111/j.1742-1241.2007.01437.x

[9] Abbatecola, A.M., Maggi, S. and Paolisso, G. (2008) New Approaches to Treating Type 2 Diabetes Mellitus in the Elderly: Role of Incretin Therapies. Drugs Aging, 25, 913-925. http://dx.doi.org/10.2165/0002512-200825110-00002

[10] Maeda, H., Kubota, A., Tanaka, Y., Terauchi, Y. and Matsuba, I. (2012) The Safety, Efficacy and Predictors for HbA1c Reduction of Sitagliptin in the Treatment of Japanese Type 2 Diabetes. Diabetes Research and Clinical Practice, 95, e20-e22. http://dx.doi.org/10.1016/j.diabres.2011.10.011

[11] Kubota, A., Maeda, H., Kanamori, A., Matoba, K., Jin, Y., Minagawa, F., Obana, M., et al. (2012) Efficacy and Safety of Sitagliptin Monotherapy and Combination Therapy in Japanese Type 2 Diabetes Patients. Journal of Diabetes Investigation, 3, 503-509. http://dx.doi.org/10.1111/j.2040-1124.2012.00221.X

[12] Kubota, A., Matsuba, I., Saito, T., Nabe, K. and Seino, Y. (2011) Secretory Units of Islets in Transplantation Index Is a Useful Clinical Marker to Evaluate the Efficacy of Sitagliptin in Treatment of Type 2 Diabetes Mellitus. Journal of Diabetes Investigation, 2, 377-380. http://dx.doi.org/10.1111/j.2040-1124.2011.00109.x

[13] Kubota, A., Maeda, H., Kanamori, A., Matoba, K., Jin, Y., Minagawa, F., Obana, M., et al. (2012) Pleiotropic Effects of Sitagliptin in the Treatment of Type 2 Diabetes Mellitus Patients. Journal of Clinical Medicine Research, 4, 309-313. http://dx.doi.org/10.4021/jocmr1061w

[14] Chung, H.S. and Lee, M.K. (2011) Efficacy of Sitagliptin When Added to Ongoing Therapy in Korean Subjects with Type 2 Diabetes Mellitus. Diabetes \& Metabolism Journal, 35, 411-417. http://dx.doi.org/10.4093/dmj.2011.35.4.411

[15] Nonaka, K., Kakikawa, T., Sato, A., Okuyama, K., Fujimoto, G., Kato, N., Suzuki, H., et al. (2008) Efficacy and Safety of Sitagliptin Monotherapy in Japanese Patients with Type 2 Diabetes. Diabetes Research and Clinical Practice, 79, 291-298. http://dx.doi.org/10.1016/j.diabres.2007.08.021

[16] Tajima, N., Kadowaki, T., Odawara, M., Nishii, M., Taniguchi, T. and Arjona Ferreira, J.C. (2011) Addition of Sitagliptin to Ongoing Glimepiride Therapy in Japanese Patients with Type 2 Diabetes over 52 Weeks Leads to Improved Glycemic Control. Diabetology International, 2, 32-44. http://dx.doi.org/10.1007/s13340-011-0022-2

[17] Kashiwagi, A., Kadowaki, T., Tajima, N., Nonaka, K., Taniguchi, T., Nishii, M., Arjona Ferreira, J.C. and Amatruda, J.M. (2011) Sitagliptin Added to Treatment with Ongoing Pioglitazone for Up to 52 Weeks Improves Glycemic Control in Japanese Patients with Type 2 Diabetes. Journal of Diabetes Investigation, 2, 381-390. 
http://dx.doi.org/10.1111/j.2040-1124.2011.00120.x

[18] Kadowaki, T., Tajima, N., Odawara, M., Nishii, M., Taniguchi, T. and Arjona Ferreira, J.C. (2013) Addition of Sitagliptin to Ongoing Metformin Monotherapy Improves Glycemic Control in Japanese Patients with Type 2 Diabetes over 52 Weeks. Journal of Diabetes Investigation, 42, 174-181. http://dx.doi.org/10.1111/jdi.12001

[19] Tajima, N., Kadowaki, T., Okamoto, T., Sato, A., Okuyama, K., Minamide, T. and Arjona Ferreira, J.C. (2013) Sitagliptin Added to Voglibose Monotherapy Improves Glycemic Control in Patients with Type 2 Diabetes. Journal of Diabetes Investigation, 4, 595-604.

[20] Kadowaki, T., Tajima, N., Odawara, M., Minamide, T., Kawashima, M., Yanagida, D., Okamoto, T. and Arjona Ferreira, J.C. (2013) Efficacy and Safety of Sitagliptin Add-On Therapy in Japanese Patients with Type 2 Diabetes on Insulin Monotherapy. Diabetology International, 4, 160-172. http://dx.doi.org/10.1007/s13340-013-0109-z

[21] Johnston, S.S., Conner, C., et al. (2012) Association between Hypoglycaemic Events and Fall-Related Fractures in Medicare-Covered Patients with Type 2 Diabetes. Diabetes, Obesity and Metabolism, 14, 634-643. http://dx.doi.org/10.1111/j.1463-1326.2012.01583.x

[22] Whitmer, R.A., Karter, A.J., et al. (2009) Hypoglycemic Episodes and Risk of Dementia in Older Patients with Type 2 Diabetes Mellitus. JAMA, 301, 1565-1572. http://dx.doi.org/10.1001/jama.2009.460

[23] Bonds, D.E., Miller, M.E., et al. (2010) The Association between Symptomatic Severe Hypoglycemia and Mortality in Type 2 Diabetes: Retrospective Epidemiological Analysis of the ACCORD Study. BMJ, 340, b4909. http://dx.doi.org/10.1136/bmj.b4909

[24] Iwakura, T., Sasaki, S., et al. (2012) Clinical Analysis of 135 Type 2 Diabetes Patients with Severe Drug-Induced Hypoglycemia. Journal of the Japan Diabetes Society, 55, 857-865.

[25] Pratley, R.E., Rosenstock, J., et al. (2007) Management of Type 2 Diabetes in Treat-Naïve Elderly Patients: Benefits and Risks of Vildagliptin Monotherapy. Diabetes Care, 30, 3017-3022. http://dx.doi.org/10.2337/dc07-1188

[26] Schweizer, A., Dejager, S., et al. (2011) Clinical Experience with Vidagliptin in the Management of Type 2 Diabetes in a Patient Population $\geq 75$ Years: A Pooled Analysis from a Database of Clinical Trials. Diabetes, Obesity and Metabolism, 13, 55-64. http://dx.doi.org/10.1111/j.1463-1326.2010.01325.x

[27] Barnett, A.H., Huisman, H., et al. (2013) Linagliptin for Patients Aged 70 Years or Older with Type 2 Diabetes Inadequately Controlled with Common Anti-Diabetes Treatments: A Randomized, Double-Blind, Placebo-Controlled Trials. The Lancet, 382, 1413-1423. http://dx.doi.org/10.1016/S0140-6736(13)61500-7 